\title{
Forecast: Program to obtain forecasts from subjects for successive values of chaotic time series
}

\author{
MARY ANN METZGER and MICHAEL F. THEISZ \\ University of Maryland UMBC, Baltimore, Maryland
}

\begin{abstract}
The program Forecast conducts a session to obtain forecasts from subjects for successive values of an evolving chaotic time series. The user of Forecast can control the session by selecting a number of options for the time series, including both the type (Hénon, or logistic) and degree of chaos. Forecast was created in Turbo Pascal and has been run on a 386 AT with a mouse and on a PS/2 with either a mouse or a touch-sensitive screen. Single sessions conducted by Forecast have collected 600 forecasts at a rate of one per $2 \mathrm{sec}$ from adults and, without alteration, 180 forecasts from young (3-5 years) children. Compared with three procedures using other display modes or response modes, Forecast collected about three times the number of forecasts in sessions that were only about a third to one half as long.
\end{abstract}

Forecast conducts a session to obtain forecasts from subjects for successive values of an evolving chaotic time series. A time series is a sequence of numbers that can be interpreted as a sequence of observations on a process. Forecast has obtained 600 consecutive forecasts from adults at a rate of one forecast per $2 \mathrm{sec}$. For each session, Forecast presents instructions to an individual subject via video display, generates the time series selected by the user, generates spatial displays of the time series, conducts practice trials, translates mouse movements into numerical forecasts, and records results in a data file. The procedure used for adult subjects has been used without alteration for young (3-5 years) children. The program was designed to overcome problems associated with subject fatigue, which have previously limited the number of consecutive forecasts that could be obtained.

\section{Previous Theory and Data on Forecasting}

The study of forecasting per se is relatively new, but it follows from the tradition of experiments on probability matching and judgmental heuristics. In these areas of research, subjects are asked to estimate uncertain quantities, and their estimates are compared with a normative model. The practical field of economic forecasting,

Support from NSF Grant BNS-9020357 is acknowledged. The source code forecast.pas is available via anonymous ftp from archive.cs.umbc.edu, subdirectory pub, subdirectory niccolo, file t930402.pas. Operating instructions are in file t930403.txt. To obtain this information by mail, send one 3.5 -in. formatted, high-density diskette to the address below. Include a self-addressed diskette mailer. The authors thank the staff at the computer center at UMBC for technical assistance and support. Send electronic requests and inquiries to metzger@umbc.edu. Send other inquiries to M. A. Metzger, Department of Psychology, University of Maryland UMBC, Baltimore, MD 21228. combined with the study of deterministic chaos, has led to several normative theories of forecasting, including approaches based on methods of (1) linear time series (Makridakis et al., 1984); (2) dynamic models and Bayesian forecasting (West \& Harrison, 1989); (3) connectionist forecasting (Weigend, Huberman, \& Rumelhart, 1990); and (4) dynamical systems methods (Lorenz, 1989; Parker \& Chua, 1987; Shaw, 1984). Empirical studies of forecasting appear in literature regarding the practical field of business management-for example, a study of managers operating simulated, complex industrial production plants (Sterman, 1989)and to theories of cognition and behavior (Metzger, 1994, in press; Neuringer \& Voss, 1993).

\section{Terminology and Principles}

Definitions. The properties of chaos that are relevant here are those directly related to forecasting. Townsend (1992) gives a discussion of other distinctive properties of chaos. The vocabulary and concepts associated with mathematical representation of a dynamical process concern the temporal patterns of values of a variable, $X$, which characterizes the state of the process. The systematic change in $X$ is usually expressed in a difference equation, which specifies how to calculate the next value, $X_{t+1}$, from the current value, $X_{t}$. Fundamental characteristics of the process are related to its linearity or nonlinearity; the system is linear if only first-degree terms of $X$ appear in the difference equation. In the processes to be considered here, the difference equation is quadratic in $X$, making the process nonlinear.

For forecasting future values of a nonlinear process, the important features of the system are the attractors; an attractor consists of a limited set of the possible values of $X_{t}$. The set of particular values of $X_{t}$ that constitute the attractor is called an invariant set, which may be 
a single asymptotic value, a cycle through a limited set of values, or another type of deterministic pattern (chaos) that may be difficult to distinguish empirically from random behavior. The salient feature of the invariant set is that the process is attracted to it. That is, whenever the system is disturbed ( $X_{t}$ is set to a value that is not in the invariant set constituting the given attractor), the disturbance lasts only a short while; soon the system returns to one of its attractors via a transient sequence of values.

Since a dynamic process tends to remain in, or quickly return to, an attractor, the attractors appear as stable states in the phenomenology of the process. While a process is in a stable state, it is said to be in the regime of the corresponding attractor. Since a given regime tends to persist, but transients are rapid, the process is unlikely to be observed in a transient, but is likely to be observed in an attractor. Transients are of interest in that they can be studied to determine the nature of the underlying process. However, when forecasting future values of $X_{t}$ for a process for which the true nature is unknown, primary interest centers on the attractors that constitute the phenomenology of the process.

Principles of long-term forecasting. The feasibility of forecasting future states of a process is limited when the process is subject to chaos, a condition for which similar initial conditions lead to dissimilar subsequent conditions. In chaos, small differences in initial conditions might send neighboring starting values of $X$ to substantially different paths. The divergence of the paths of neighboring points increases exponentially with subsequent iterations. Factors affecting the rate of divergence are the precision of measurement of initial conditions, the particular form of the difference equations, and the magnitudes of the parameters of the process. Therefore, unless the process is known to never be in a chaotic regime, accurate long-term forecasting is not possible, even when there are no substantial exogenous influences. Forecasts in the chaotic regime for arbitrarily close initial conditions diverge quickly. Therefore, the future behavior of the system loses its correlation with the initial conditions and predictability becomes nil.

The properties of chaotic attractors, added to the influence of exogenous factors, together lead to a conclusion that is important in the logic of studying one-stepahead forecasting rather than longer term forecasting. The conclusion applies to complex dynamic processes under naturally varying conditions: For nonlinear deterministic processes subject to chaos, forecasting future values of the variables of the system from past or current observations is, in general, not possible beyond the short term.

\section{TESTS OF DISPLAYS AND RESPONSE MODES}

In developing the method used by Forecast, we were first interested in investigating whether subjects forecasting a chaotic time series would perform better than subjects forecasting a similar, random time series. For each forecast, the subjects inspected the previous observations and made a one-step-ahead forecast to the next observation. Since the chaotic process generates series of numbers that are difficult to distinguish from a purely random process, a demonstration of relatively good performance on the chaotic, compared with random, time series would show that subjects could distinguish chaos from randomness, thereby justifying further study of forecasting chaotic systems. Therefore, equations of a chaotic process were used to generate successive observations for an objective chaotic time series (chaotic series), and a pseudorandom-number generator was used to generate a comparable random time series (random series). The time series generated by these two methods were used in the initial experiments that compared methods for obtaining forecasts from subjects. Thereafter, we tested changes in the program intended to improve the method of collecting forecasts.

\section{METHOD}

\section{Subjects}

The adult subjects were volunteers satisfying a course requirement in introductory psychology at UMBC. The child subjects all attended the Christian Day Care Center in Baltimore. The characteristics of the subjects for each type of procedure are given in Table 2 .

\section{Procedure}

In developing Forecast, different types of display and response modes were evaluated. The procedures that involved responses via a mouse were retained in the final version of Forecast. The final version will also operate without alteration, accepting responses to a touch-sensitive screen. Five types of procedures were tested; in each, separate groups of subjects made only one type of forecast in only one condition.

1. NS-many. The time series was displayed on the screen in either a numerical (Group N) or a spatial (Group S) display. For the numerical display, 59 consecutive, three-digit numerical values of the objective time series were written consecutively on 10 lines of a single screen. The 59 numbers remained on the screen until the subject typed in a number forecasting the 60 th value. For the spatial display, the numbers in the objective time series were translated into one of 26 spatial positions, identified by the letters of the alphabet. Each value was displayed as a blank position in an otherwise alphabetically displayed line of 26 letters. Fifty-nine such lines of letters were displayed at a rate of 1200 baud. The display then stopped, with the most recent 20 lines visible, until the subject typed in a letter forecasting the position of the blank for the 60 th line. After each forecast, the subject was shown the correct value or position of the blank. For NS-many, the subject viewed 10,800 (many) values, forecasting every 60 th value by typing three-digit numbers or 1 of 26 letters on the keyboard.

2. NS-few. The display was similar to NS-many, except that each subject viewed only 200 (few) values, forecasting each consecutive value as the series evolved.

3. TSG-key. The time series was displayed as an evolving conventional time series graph (TSG); examples are given in Figure 1. The rate at which the values were displayed depended on the subject. For TSG-key, the subject viewed 300 values, forecasting each consecutive value by using the up and down arrows to place the cursor at the vertical position corresponding to his or her forecast and then pressing the enter key.

4. TSG/1-mouse. The time series was displayed as those in TSG-key, except the rate at which the values were displayed was 

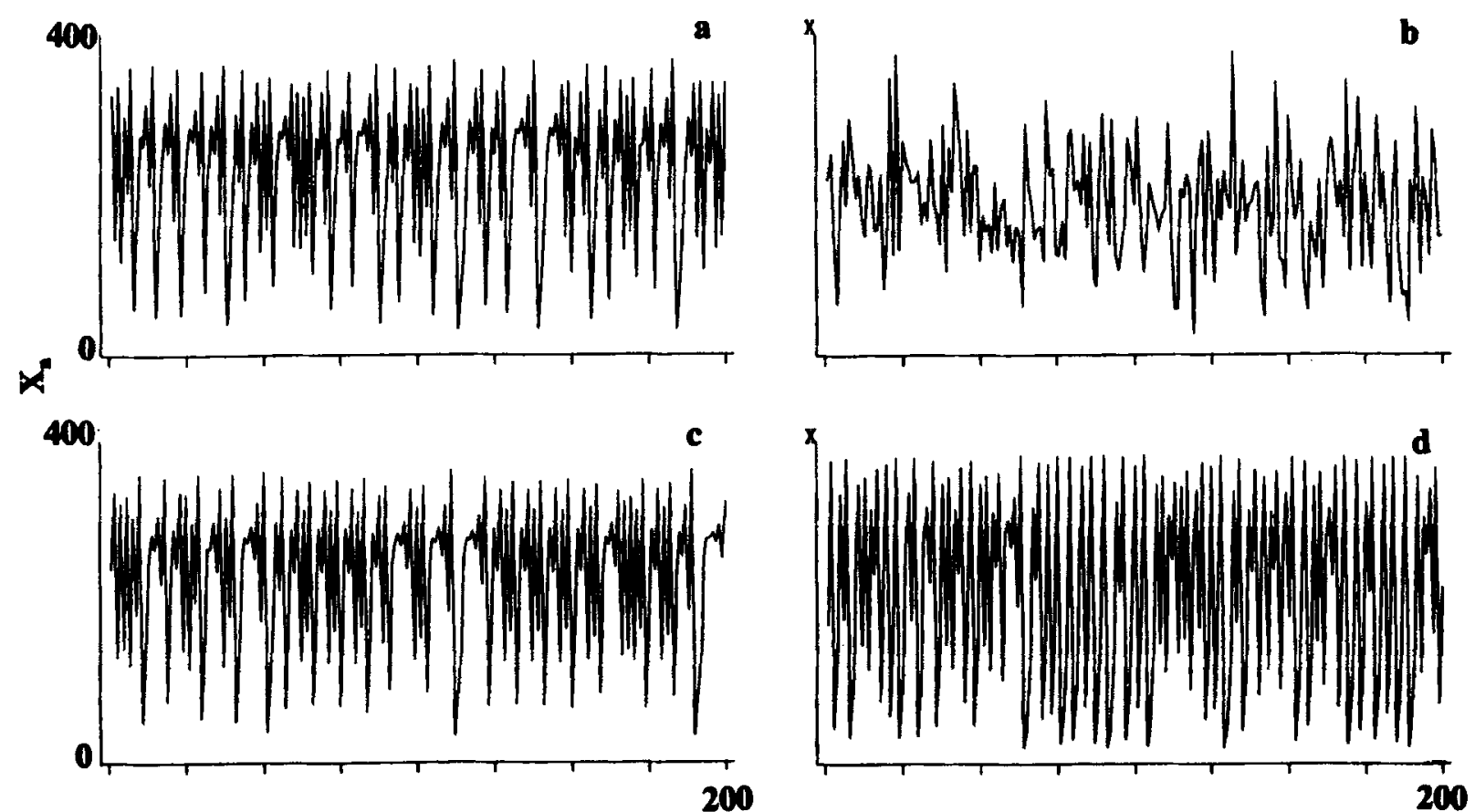

$\mathbf{n}$

Figure 1. Time series graphs for 200 observations. (a) Chaotic series, from Equations 4 and 5 , where $a=1.4$ and $b=0.3$. (b) Random series, normal distribution. (c) Hénon series from Equations 1 and 2, where $a=1.4$ and $b=0.3$. (d) Logistic series from Equation 3, where $a=3.98$.

preset at $1 \mathrm{sec}$ per value, with no breaks or pauses. The subjects forecast 600 consecutive values. For TSG/1-mouse, the subject moved the mouse cursor to the vertical position corresponding to his or her forecast. Only vertical components of mouse movement were interpreted and displayed on the screen.

5. TSG/2-mouse. The display and response modes were the same as those in TSG/1-mouse, except the rate of display was preset at 2 sec per value. The adults forecast 600 consecutive values; the children forecast 180 .

In NS-many and NS-few, forecasts were obtained, without pretraining, from separate groups of subjects for the chaotic series and the random series. For the remaining methods, forecasts were obtained only for the chaotic series.

The chaotic series. To permit study of a system in a chaotic attractor for an extended period of time, observations on a chaotic process were generated by using equations that were variants ${ }^{1}$ of Equations 1 and 2, which define the Hénon difference equation in standard form with parameters $a$ and $b$. Equations 1 and 2 were retained for the final version of Forecast, but the variant was used for all of the experiments reported here. Under consideration here is the temporal series of observations that may be represented (as in Figure 1) by a graph of $X_{t}$ against $t$, which is the iteration number that may be considered to be discrete time. The values of $X_{t}$ were generated by 10,800 iterations of the variants of Equations 1 and 2 , beginning with $Y_{0}=.2$ and $X_{0}=.1$. The transient values $X_{0}$ through $X_{99}$ were dropped from the analysis. To avoid presenting negative or decimal numbers to the subject, each value was multiplied by 100 , added to 200 , and rounded to the nearest integer.

$$
\begin{gathered}
X_{t+1}=1-a X_{t}^{2}+Y_{t} \\
Y_{t+1}=b X_{t}
\end{gathered}
$$

The 10,800 values $\left\{X_{t}\right\}$ were generated for the chaotic time series with the parameters $a=1.4$ and $b=0.3$. The values represented observations on the system over time, each set in the regime of a chaotic attractor. The histogram for the chaotic series is depicted in Figure 2. The distribution had a mean of 335, a standard deviation of 101 , and quartiles of 252,356 , and 417 .

The random series. The distribution of values of the chaotic series can be compared with the distribution of values from the random series. The random series consisted of 10,800 values randomly sampled via a pseudorandom-number generator (function RNOR from FORTRAN library CMLIB), from a normal distribution with a specified mean of 335 and a standard deviation of 101. The statistics for the random series thus generated were a mean of 333 , a standard deviation of 101 , and quartiles of 265 , 333 , and 401 . The histogram for the random series is depicted in Figure 3.

Comparison with formulas for forecasting. For comparison with the forecasting performance of subjects, the forecasting performance of two types of mathematical formulas are presented in Table 1. Each formula produced one-step-ahead forecasts based on the weighted averages of the most recent observations. One type of formula was a moving average; the other type was an autoregressive linear forecasting formula. 


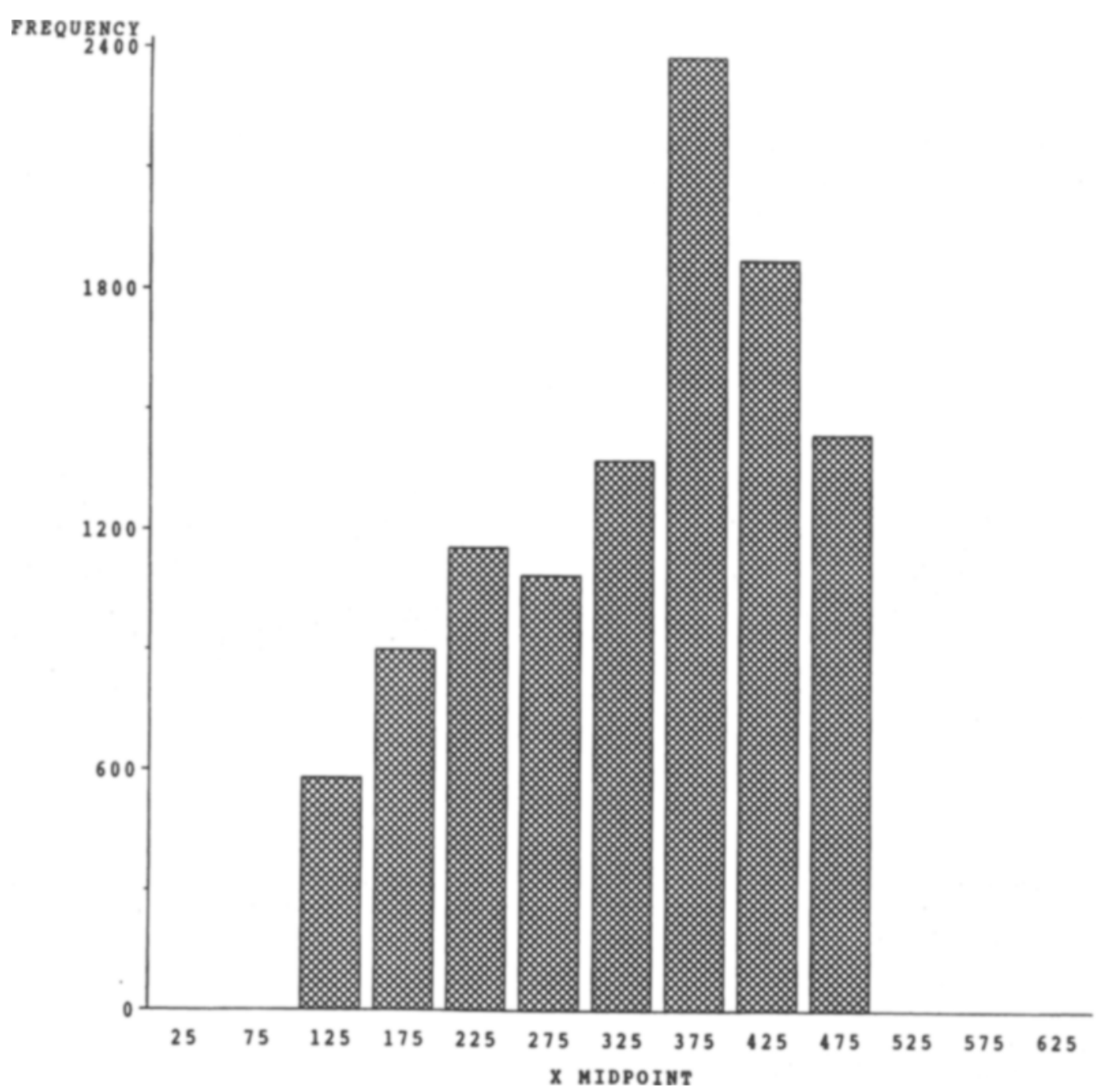

Figure 2. Histogram for the distribution of values in the chaotic series.

The moving-average formula was used to generate six sets of forecasts. For each value of $k(k=1,3,7,10,100$, and 1,000$)$, the formula made a forecast for each possible equilibrium value from the mean of the $k$ immediately preceding points. In the case of the random sample, applying the moving-average formula was not conceptually different from estimating the mean from a random sample of size $k$, the properties of which are known analytically. Separate sets of forecasts for each time series, chaotic and random, were obtained for each value of $k$.

A best linear predictor was obtained for each time series. The coefficients for the predictor were determined with the restriction that the prediction be made from the most recent observation $\left(A_{t}\right)$ and the most recent first- and second-order differences $\left(D 1_{t}=\right.$ $A_{t}-A_{t-1}$ and $D 2_{t}=D 1_{t}-D 1_{t-1}$, respectively). These three quantities are analogous to the current position, velocity, and acceleration of the time series. The problem was stated in this way to capture any local patterns based on rates of change and differences in rates of change that might be useful in one-step-ahead forecasting. Other analyses showed that the predictor, thus restricted, performed almost identically to the best linear predictor (which settled on the preceding eight observations) selected as optimal according to the Akaike Information Criterion (Akaike, 1974).

For the chaotic sequence, the coefficients for $A, D 1$, and $D 2$, respectively, were $-.44, .54$, and -.31 ( $p=.000$, for each). These coefficients for the chaotic time series were large and reliable enough to confer an advantage for the linear predictor over the moving averages.

For the random time series, the transition coefficients for $A, D I$, and $D 2$, respectively, were $.01,-.01$, and $-.01(p=.000$, for each). Although the coefficients for the random series are significantly nonzero, perhaps because of small dependencies in the pseudorandom-number generator acting over 10,800 observations, the coefficients are small enough that the forecast is always, as would be expected, very nearly the mean. Thus, for the random series, the linear predictor has no advantage over a moving average of large $k$.

\section{RESULTS AND DISCUSSION}

Sessions for the first three experiments were conducted by precursors of Forecast, and they had the following durations: NS-many ranged from $45-90 \mathrm{~min}$, NS-few from $30-45 \mathrm{~min}$, and TSG-key was $30 \mathrm{~min}$. For these procedures, most of the subjects spontaneously mentioned that the session was tiring. Because of both session length and literacy requirements, these three 


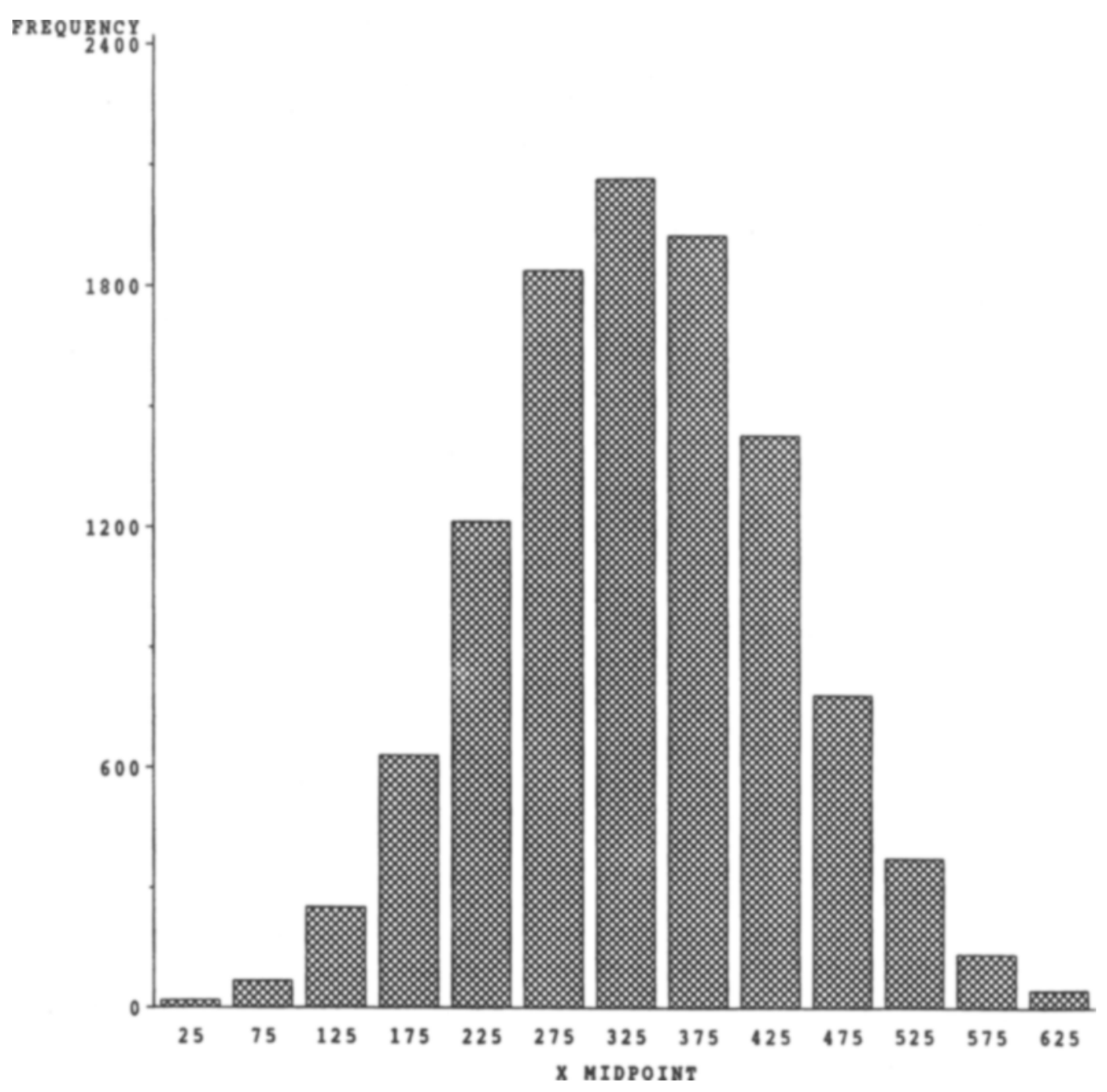

Figure 3. Histogram for the distribution of values in the random series.

procedures were obviously unsuitable for young children. Sessions for the remaining two experiments were conducted by Forecast and had the following durations: TSG/1-mouse ranged from $12-15 \mathrm{~min}$, and TSG/2mouse ranged from $22-25 \mathrm{~min}$ (adults) or 8-10 min (children).

The aim of the NS-many condition was to determine whether the subjects, given extensive exposure to the chaotic series, could make accurate one-step-ahead forecasts. In terms of quartiles of distribution of errors, defined as the absolute value of the discrepancy between the subject's forecast and the objective value of the time series, the subject's accuracy is presented in Table 2 . In terms of correlations between forecasts and the chaotic or random time series, the accuracy is presented in Table 3. The primary substantive findings shown in Tables 2 and 3 are that the subjects forecasting the chaotic series in the NS-many condition all performed about equally well-better than a moving average of any size $k$, equal to or perhaps somewhat better than the corre- sponding best linear predictor, and better than the subjects forecasting the corresponding random series.

The results for NS-many showed that the subjects were able to make accurate forecasts, but since the forecasts were not for consecutive values and were sparse compared with the number of values inspected, details of the learning process were not evident. In addition, the extensive exposure required to obtain 180 forecasts resulted in long sessions with evidence of fatigue. NS-few was therefore devised in order to obtain 200 consecutive forecasts. The results of NS-few showed that 200 was an insufficient number of trials to bring forecasting accuracy to the level shown by the subjects in NS-many. On the assumption that forecasting accuracy could develop after viewing somewhere between 200 and 10,800 values of the time series, the procedure was then changed to permit more than 200 consecutive forecasts to be examined within a single session. This resulted in the TSG-key procedure, in which 300 consecutive values were displayed as points on an evolving time series 
Table 1

Distribution of Absolute Errors in One-Step-Ahead Forecasts Generated From Two Types of Mathematical Forecasting Formulas

\begin{tabular}{|c|c|c|c|c|c|c|}
\hline \multirow[b]{2}{*}{ Method } & \multirow[b]{2}{*}{$k$} & \multirow{2}{*}{$\begin{array}{c}\text { No. of } \\
\text { Forecasts }\end{array}$} & \multirow{2}{*}{$\begin{array}{c}\text { Mean Absolute } \\
\text { Errors }\end{array}$} & \multicolumn{3}{|c|}{ Percentile } \\
\hline & & & & 25 & 50 & 75 \\
\hline \multicolumn{7}{|c|}{ Forecasting the Chaotic Series } \\
\hline \multirow[t]{2}{*}{ Moving average } & 1 & 10,799 & 138 & 66 & 118 & 199 \\
\hline & & 180 & 127 & 60 & 112 & 188 \\
\hline \multirow[t]{2}{*}{ Moving average } & 3 & 10,797 & 107 & 59 & 97 & 145 \\
\hline & & 180 & 97 & 54 & 90 & 126 \\
\hline \multirow[t]{2}{*}{ Moving average } & 7 & 10,793 & 92 & 48 & 88 & 127 \\
\hline & & 180 & 84 & 38 & 83 & 114 \\
\hline \multirow[t]{2}{*}{ Moving average } & 10 & 10,790 & 93 & 45 & 88 & 126 \\
\hline & & 180 & 84 & 43 & 85 & 118 \\
\hline \multirow[t]{2}{*}{ Moving average } & 100 & 10,700 & 86 & 43 & 83 & 123 \\
\hline & & 179 & 82 & 45 & 84 & 113 \\
\hline \multirow[t]{2}{*}{ Moving average } & 1,000 & 9,800 & 86 & 43 & 82 & 123 \\
\hline & & 164 & 82 & 43 & 82 & 115 \\
\hline \multirow[t]{2}{*}{ Linear predictor } & & 10,797 & 75 & 31 & 72 & 109 \\
\hline & & 180 & 75 & 35 & 73 & 108 \\
\hline \multicolumn{7}{|c|}{ Forecasting the Random Series } \\
\hline \multirow[t]{2}{*}{ Moving average } & 1 & 10,799 & 113 & 45 & 95 & 167 \\
\hline & & 180 & 121 & 36 & 106 & 180 \\
\hline \multirow[t]{2}{*}{ Moving average } & 3 & 10,797 & 92 & 37 & 78 & 133 \\
\hline & & 180 & 95 & 38 & 78 & 145 \\
\hline \multirow[t]{2}{*}{ Moving average } & 7 & 10,793 & 86 & 35 & 73 & 122 \\
\hline & & 180 & 90 & 35 & 79 & 130 \\
\hline \multirow[t]{2}{*}{ Moving average } & 10 & 10,790 & 84 & 34 & 71 & 121 \\
\hline & & 180 & 87 & 35 & 67 & 130 \\
\hline \multirow[t]{2}{*}{ Moving average } & 100 & 10,700 & 81 & 33 & 68 & 116 \\
\hline & & 179 & 85 & 31 & 75 & 122 \\
\hline \multirow[t]{2}{*}{ Moving average } & 1,000 & 9,800 & 81 & 33 & 68 & 115 \\
\hline & & 164 & 86 & 32 & 76 & 121 \\
\hline \multirow[t]{2}{*}{ Linear predictor } & & 10,797 & 80 & 33 & 68 & 115 \\
\hline & & 180 & 84 & 31 & 73 & 118 \\
\hline
\end{tabular}

Note-Two lines are given for each forecasting formula. The first line gives the performance of the formula over all observations; the second line gives the performance of the formula on the specific observations forecasted by subjects in NS-many.

graph with subjects registering their forecast via arrow keys. The TSG-key procedure resulted in an increase in accuracy of forecasting, but it did not bring accuracy up to the level of performance displayed by the subjects with extensive experience. Hence, in order to study the details of the acquisition of accurate forecasting, we needed a method to obtain consecutive forecasts of somewhere between 300 and 10,800 consecutive values. Forecast, used to conduct the sessions of TSG/1-mouse and TSG/2-mouse, was developed to increase the number of consecutive forecasts that could be obtained within a single session.

For TSG/2-mouse, the subjects saw only 600 consecutive values of the time series and made 600 forecasts, but their accuracy was similar to that of the subjects in NS-many, as is shown in Tables 2 and 3. The increased efficiency of the session run by Forecast permitted the procedure that was used for the adults to be used without modification for the 3- to 5-year-old children, also shown in Tables 2 and 3, from whom 180 consecutive forecasts could be obtained.

Table 3 shows that not all the children responded at random. One third had significant negative or positive correlations between their forecasts and the objective series. If the children had responded at random, only about $5 \%$ of the correlations would be expected to be significantly different from zero. There were seven significant positive correlations, ranging from .21 to .47 , and six negative, -.15 to -.27 . These findings are similar to those for adult performance on NS-few, for which two of five correlations were significantly different from zero, -.15 and -.31 . Both positive and negative correlations show systematic responding. Therefore, the children were not only tolerating the procedure, they were also, at least sometimes, responding systematically with respect to forecasting.

\section{PROGRAM DESCRIPTION}

Created in Turbo Pascal, Forecast has been run on a 386 AT with VGA and mouse, and on a PS/ 2 using either VGA and mouse or an IBM 8614 VGA with a touch-sensitive screen. The user of Forecast should be familiar with creating text files and directories. At the beginning of each session, Forecast prompts the user for the identification number of the subject and the location of a user-created text file containing specifications for the experimental session. The parameters and options include experiment number; type of chaotic process;

Table 2

Distribution of Absolute Errors Made by Subjects for Three Types of Display-Response Modes for the Chaotic or Random Series

\begin{tabular}{lccrrrrr}
\hline & & & \multirow{2}{*}{$\begin{array}{c}\text { No. } \\
\text { Method }\end{array}$} & Series & Subjects & No. of & \multicolumn{3}{c}{ Quartiles } \\
\cline { 6 - 8 } & Viewed & Forecasts & 25 & 50 & 75 \\
\hline NS-many & chaotic & 8 adult F & 10,800 & 180 & 20 & 56 & 117 \\
NS-few & chaotic & 5 adult F & 200 & 200 & 50 & 106 & 187 \\
TSG-key & chaotic & 6 adult MF & 300 & 300 & 36 & 92 & 172 \\
TSG/1-mouse & chaotic & 7 adult MF & 600 & 600 & 36 & 80 & 148 \\
TSG/2-mouse & chaotic & 7 adult MF & 600 & 600 & 27 & 59 & 112 \\
TSG/2-mouse & chaotic & 40 child MF & 180 & 180 & 53 & 115 & 197 \\
NS-many & random & 8 adult F & 10,800 & 180 & 46 & 88 & 161 \\
NS-few & random & 5 adult F & 200 & 200 & 46 & 96 & 172 \\
\hline
\end{tabular}

Note-Quartiles are aggregated over subjects. Children were 3-5 years old. M, male; F, female; MF, both males and females. 
Table 3

Accuracy Measured by Mean Absolute Errors and Individual Correlations Between Forecasts and Objective Values of the Chaotic or Random Series

\begin{tabular}{lccrrr} 
& & & Mean Absolute & \multicolumn{2}{c}{ Correlation } \\
\cline { 5 - 6 } Method & Series & Subjects & Errors & Median & Range \\
\hline NS-many & chaotic & 8 adult F & 74 & .46 & $-.06-.76$ \\
NS-few & chaotic & 5 adult F & 124 & -.08 & $-.31-.02^{*}$ \\
TSG-key & chaotic & 6 adult MF & 116 & .22 & $.04-.32$ \\
TSG/1-mouse & chaotic & 7 adult MF & 102 & .16 & $.02-.39$ \\
TSG/2-mouse & chaotic & 7 adult MF & 80 & .40 & $.00-.58$ \\
TSG/2-mouse & chaotic & 40 child MF & 132 & .00 & $-.27-.47^{*}$ \\
NS-many & random & 8 adult F & 114 & .07 & $-.03-.13$ \\
NS-few & random & 5 adult F & 117 & -.02 & $-.12-.07$ \\
\hline
\end{tabular}

Note-M, male; F, female; MF, both males and females. *The medians in these cases do not give an accurate picture of the amount of systematic responding. Two of the 5 adults had statistically significant (two-tailed, $p<.05$ ) negative correlations. Twelve of the 40 children had significant positive correlations, and 6 had significant negative correlations.

coefficients for the chaotic equations; location of the file that will contain the output data; number of test trials; number of practice trials; option to save or discard results of practice trials; option for subject-paced or computer-paced trials; intertrial interval (seconds) for computer-paced trials; instructions (four lines) to appear on the "welcome" screen that the subject views at the beginning of the session.

After conducting practice trials, Forecast creates the display and collects responses for an experimental session. For each forecast, the subject aims the mouse cursor at a place on the screen where he or she anticipates the next point, $X_{t}$, will occur. When the computer-paced option has been selected, the position of the pointer at the end of the intertrial interval is taken to be the forecast. When the subject-paced option has been selected, the position of the pointer at the time of the mouse click is taken. The horizontal coordinate of the cursor is constrained by the program in such a way that the cursor is permitted to move only along an undisplayed vertical line. At the end of the trial, the coordinates of the mouse cursor are recorded, the vertical coordinate of which will later be converted to a numerical value defined as the forecast $\left(F_{t}\right)$ for time $t$. The error for time $t$ will then be calculated as $E_{t}=X_{t}-F_{t}$. After the coordinates of the cursor have been recorded, the correct value, $X_{t}$, is displayed as a small dot located on the graph at $\left(X_{t}, t\right)$. The point representing $X_{t}$ is then connected by a straight line to the point representing $X_{t-1}$.

The resulting display eventually covers the screen from left to right with the connected graph of $X_{t}$ against $t$, with an optional number of observations per screen. When the right end of the screen is reached, the screen is cleared and the graph resumes from the left side of the screen. At the end of each session, the results are recorded in an output file. Each line of the file contains the experiment number and the subject number, $X_{t}, E_{t}$, and $t$.

The user may select the Hénon system given in Equations 1 and 2, or the logistic system given in Equation 3 (May, 1976). In addition, the user may determine the de- gree of chaos in the time series by specifying values for the parameters $a$ and/or $b$ of the equations.

$$
X_{t+1}=a X_{t}\left(1-X_{t}\right)
$$

The program uses DOS, Graph, and Crt, which are standard Turbo Pascal options. It also uses GraphMouseU, an adaptation of MouseU.pas (Crosbie, 1990). GraphMouseU is distributed with Forecast.

See the acknowledgments for instructions on how to obtain operating instructions, source, and executable versions of Forecast via ftp or on diskette.

\section{REFERENCES}

AKAIKE, H. (1974). Markovian representation of stochastic processes and its application to the analysis of autoregressive moving average processes. Annals of the Institute of Statistical Mathematics, 26, 363-387.

Crosbie, J. (1990). The Microsoft mouse as a multipurpose response device for the IBM PC/XT/AT. Behavior Research Methods, Instruments, \& Computers, 22, 305-316.

Grebogi, C., OTT, E., \& Yorke, J. A. (1987). Chaos, strange attractors, and fractal basin boundaries in nonlinear dynamics. Science, 238, 632-638.

LORENZ, H. W. (1989). Nonlinear dynamical economics and chaotic motion. Berlin: Springer-Verlag.

Makridakis, S., Anderson, A., Carbone, R., Fildes, R., Hibon, M., Lewandowski, R., Newton, J., Parzen, E., \& Winkler, R. (1984). The forecasting accuracy of major time series methods. New York: Wiley.

MAY, R. M. (1976). Simple mathematical models with very complicated dynamics. Nature, 261, 459-467.

MetzGer, M. A. (1994). Have subjects been shown to generate chaotic numbers? Commentary on Neuringer and Voss. Psychological Science, 5, 111-114.

Metzger, M. A. (in press). Multi-process dynamic models applied to the dynamics of cognition and behavior. In R. Port \& T. Van Gelder (Eds.), Mind as motion: Dynamics, behavior, and cognition. Cambridge, MA: MIT Press.

NeURINGer, A., \& Voss, C. (1993). Approximating chaotic behavior. Psychological Science, 4, 113-119.

PARKer, T. S., \& ChUA, L. O. (1987). Chaos: A tutorial for engineers. Proceedings of the IEEE, 75, 983-1008.

SHAw, R. (1984). The dripping faucet as a model chaotic system. Santa Cruz, CA: Aerial.

Sterman, J. D. (1989), Deterministic chaos in an experimental eco- 
nomic system. Journal of Economic Behavior \& Organization, 12, $1-28$

TownSEND, J. T. (1992). Chaos theory: A brief tutorial and discussion. In A. F. Healy, S. M. Kosslyn, \& R. M. Shiffrin (Eds.), From learning theory to connectionist theory: Essays in honor of William $K$. Estes (Vol. 1, pp. 65-96). Hillsdale, NJ: Erlbaum.

Weigend, A. S., Huberman, B. A., \& Rumelhart, D. E. (1990). Predicting the future: A connectionist approach (Tech. Rep. No. PDP90-01). Stanford, CA: Stanford University.

WEST, M., \& HARRISON, J. (1989). Bayesian forecasting and dynamic models. New York: Springer-Verlag.

\section{NOTE}

1. The variant of the chaotic process defined by Equations 4 and 5 was used for the chaotic attractor in the experiments and data reported here. Equations 4 and 5 were taken from Grebogi, Ott, and Yorke
(1987), who analyzed their chaotic properties and identified them as the equations for the Hénon attractor. Equations 4 and 5 constitute a minor variation that is similar to the standard Henon attractor given in Equations 1 and 2 . The similarity of the time series can be seen in Figure 1 by comparing panel a with panel $\mathrm{c}$. As distributed, Forecast uses Equations 1 and 2, the standard Hénon attractor.

$$
\begin{gathered}
X_{t+1}=a-X_{t}^{2}+b Y_{t} \\
Y_{t+1}=X_{t}
\end{gathered}
$$

(Manuscript received November 4, 1992; revision accepted for publication February 16, 1994.) 